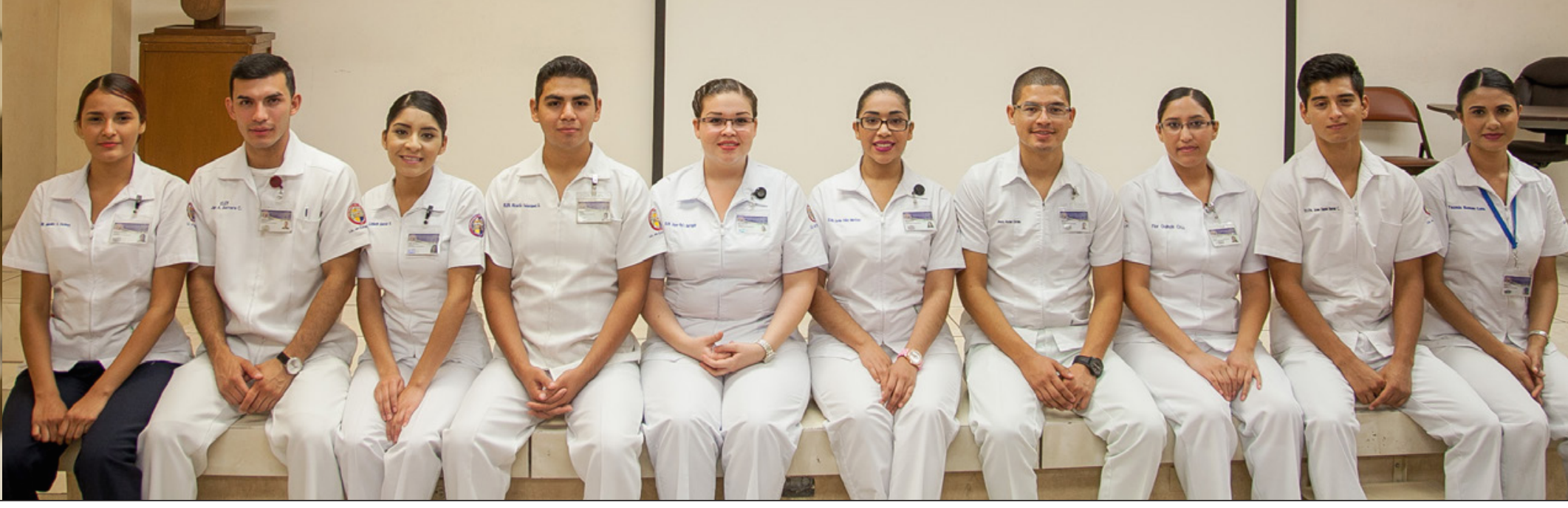

\title{
AUTOCUIDADO PRESENTE EN ESTUDIANTES DE ENFERMERÍA DE LA UNIVERSIDAD DE SONORA
}

\author{
SELF- CARE PRESENT IN NURSING STUDENTS FROM THE UNIVERSITY OF SONORA.
}

\section{ABSTRACT}

Self-care refers to the practice of activities that individuals initiate and perform to their advantage to maintain life, health and welfare. The future health professionals should be able to make a positive change in people, therefore must possess a self-care in their daily work, enabling them to live healthily and be on welfare to care for others, fulfilling the role of model and educator. This study aimed to meet self-care practices of students in the Bachelor of Nursing third semester at the University of Sonora, Central Campus. Quantitative, descriptive, prospective and transversal study. With a sample of 100 students of Nursing. Self-care of this sample resulted in a regular level. Minor self-care practices were found in the areas of sleep, rest, nutrition, physical activity, and lack of regular review of preventive health. Conclusion: The University of Sonora in conjunction with the Department of Nursing can contribute actively to improve the level of self-care youth through programs that promote healthy practices.

Keywords: Self-care, students, healthy practices.

\section{RESUMEN:}

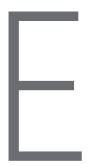
I autocuidado se refiere a la práctica de actividades que los individuos inician y realizan en su propio beneficio para el mantenimiento de la vida, la salud y el bienestar. Los futuros profesionales de la salud deben ser capaces de provocar un cambio positivo en las personas, por lo tanto deben poseer un autocuidado en su diario quehacer, que les permita vivir saludablemente y estar en bienestar para poder cuidar de otros, cumpliendo con el rol de ser modelo y educador. El presente estudio tuvo como objetivo conocer las prácticas de autocuidado de los estudiantes de la Licenciatura en Enfermería del tercer semestre de la Universidad de Sonora, Unidad Regional Centro. Estudio de tipo cuantitativo, descriptivo, prospectivo y transversal. Con una muestra de 100 de alumnos de la Lic. en Enfermería. El autocuidado de esta muestra resultó en un nivel regular. Las menores prácticas de autocuidado se encontraron en las áreas de sueño, descanso, alimentación, actividad física, y la falta de realización periódica de exámenes de salud preventivos. Conclusión: La Universidad de Sonora en conjunto con el Departamento de Enfermería puede contribuir en forma activa a mejorar el nivel de autocuidado de los jóvenes, a través de programas que impulsen prácticas saludables.

PALABRAS CLAVES: Autocuidado, estudiantes, prácticas saludables.

(*) Estudiante de Enfermería. Departamento de Enfermería. Universidad de Sonora. Email:mariana_ortegafalcon@hotmail.com
${ }^{(*)}$ Estudiante de Enfermería. Departamento de Enfermería. Universidad de Sonora. Email: jaquy_ rg28@hotmail.com
${ }^{(* * x)}$ Maestra en Ciencias. Docente de tiempo completo. Departamento de Enfermería. Universidad de Sonora. Email: Claudia_yayis@hotmail.com 
INTRODUCCIÓN

De acuerdo a la asamblea general

de las Naciones Unidas los jóvenes son personas entre los 15 y los 24 años de edad, que constituyen

el $18 \%$ de la población mundial. México es un país de jóvenes, conformando la mitad de la población total, así lo indican las estadísticas del Instituto Nacional de Estadística y Geografía (1). En una encuesta realizada por el Instituto Mexicano Nacional de la Juventud ${ }^{(2,3)}$ muestra que el $87.4 \%$ de los jóvenes evalúan positivamente su estado de salud. A pesar de esto, en el mismo estudio, el $42.4 \%$ ingieren comida rápida y

un $70 \%$ golosinas, datos que nos permiten afirmar que presentan un déficit de autocuidado.

Los futuros profesionales de la salud deben ser capaces de provocar un cambio positivo sobre la salud de las personas, por lo tanto, deben poseer un autocuidado en su diario quehacer que les permita vivir saludablemente y estar en bienestar para poder cuidar de otros.

Sin embargo, los estudiantes se encuentran en un proceso de formación en donde se convive en un ambiente que mezcla una diversidad

de costumbres, hábitos de vida y comportamientos que influyen en el cuidado de su salud a lo que se

suma la lejanía del hogar, pocos recursos económicos y la sobrecarga escolar que se traducen en restricciones para seleccionar alimentación saludable, la práctica de deportes, el

manejo del estrés, entre otras.(4)

Las conductas mencionadas anteriormente, varían de acuerdo al área de salud en las que los estudiantes se desenvuelven y a los diversos factores que afectan a sus capacidades para ocuparse de su autocuidado, planteados por Dorothea Orem $\left({ }^{5,6)}\right.$.

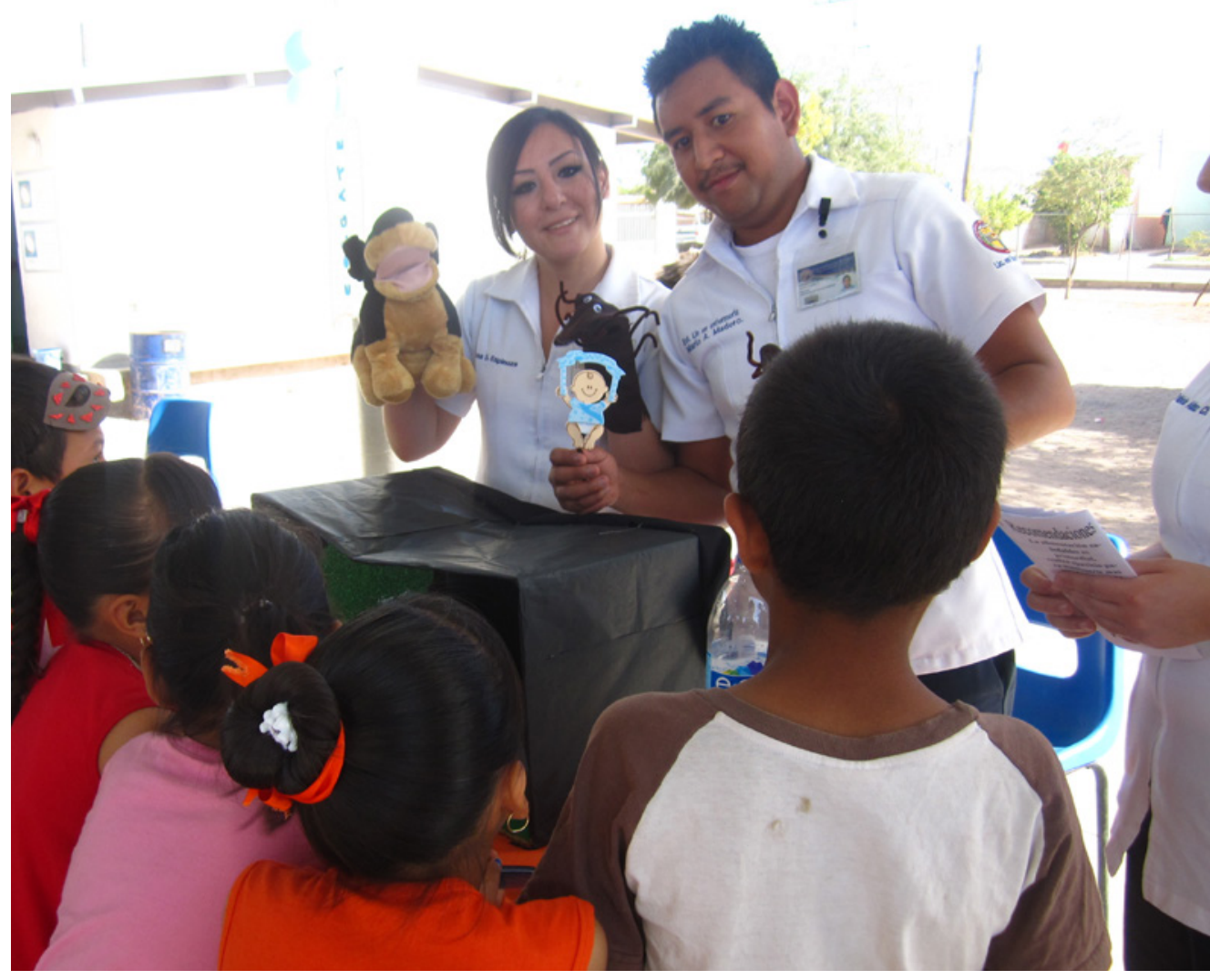

\section{METODOLOGÍA}

Para describir las prácticas de autocuidado de los estudiantes de la licenciatura de enfermería de la Universidad de Sonora, se desarrolló un estudio cuantitativo, descriptivo, prospectivo, y transversal.(7)

Para la muestra en estudio se consideró los siguientes criterios:

estudiantes de tercer semestre de la licenciatura de enfermería, debido a que son un grupo más representativo y también se consideró solo a aquellos que aceptaron participar en la investigación después de leer y firmar la carta de consentimiento informado.

La población corresponde a los alumnos de la licenciatura de enfermería de tercer semestre de la Universidad de Sonora de la Unidad Regional Centro, los cuales son 134. Se llevó a cabo un muestreo probabilístico aleatorio, conformando la muestra un total de 100 alumnos. ${ }^{(7)}$

Los datos se obtuvieron mediante una encuesta directa por medio de un cuestionario de 30 ítems escrito con respuestas dicotómicas y de opción múltiple administrada por categorías. Fue de carácter anónimo. Para validar el instrumento, se aplicó una prueba piloto a cierto número de individuos con las características correspondientes, pero que no formó parte de la muestra. ${ }^{(8)}$

Al realizar el estudio se consideraron los aspectos éticos señalados en el Reglamento de la Ley General de Salud en materia de investigación. Se proporcionó a los participantes un consentimiento informado por escrito donde, se informó el objetivo de la investigación, el procedimiento que se llevaría a cabo, la participación voluntaria y finalmente el derecho a retirar su participación en cualquier momento. La investigación no expuso riesgos ni daños a los estudiantes, debido a que el instrumento de recolección de datos fue un cuestionario por escrito totalmente confidencial. 


\section{RESULTADOS}

En referencia a la edad el $87 \%$ de la población estudiantil comprende entre los 19 y 21 años, mientras que el $13 \%$ corresponde a alumnos de 22 a 37 años. El 71\% de la población en estudio pertenecen al sexo femenino y $29 \%$ al masculino.

\section{GRAFICA 1}

Una de las preguntas formuladas, fue con el objetivo de evaluar el consumo de sustancias nocivas entre ellas el consumo de tabaco a lo que la mayoría (83\%) de los estudiantes negó consumirlo y el $17 \%$ de la población total respondió ser fumador activo. Sin embargo, se cuestionó también si evitaban espacios con humo a lo que la mayoría (54\%) mencionó que no lo evita. También se encontró que el $47 \%$ de los encuestados consume bebidas alcohólicas, mientras que el 53\% (53 personas) no consumen ningún tipo de bebidas alcohólicas.

\section{GRAFICA 2}

Para valorar hábitos de vida saludable se indagó sobre la actividad física, el $50 \%$ de la población resultó ser sedentario y el otro $50 \%$ realiza actividad física 2 o menos veces por semana. De los encuestados que respondieron no realizar ejercicio, mencionaron diversos motivos como la falta de tiempo en un $92 \%$ y la falta de interés con un $8 \%$. De igual manera se analizó la frecuencia de horas que duermen diariamente los jóvenes universitarios, obteniendo que solo el $2 \%$ duerme la cantidad adecuada de horas (más de 8 horas), un $45 \%$ más de 6 horas y el $53 \%$ no duerme lo suficiente (menos de 6 horas diarias) siendo la causa principal el cumplir con responsabilidades escolares o extraescolares (49 personas).

Una de las preguntas formuladas fue con el objetivo de valorar la frecuencia del consumo de alimentos en los

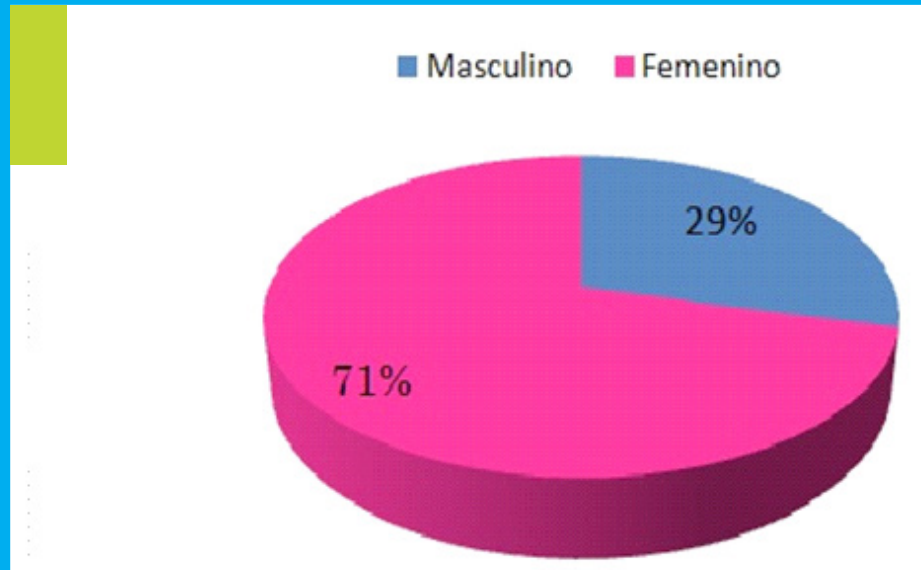

Gráfica 1 - Propoción de estudiantes de Licenciatura en Enfermería de prácticas de autocuidado ( $n=100$; fuente : encuesta directa)

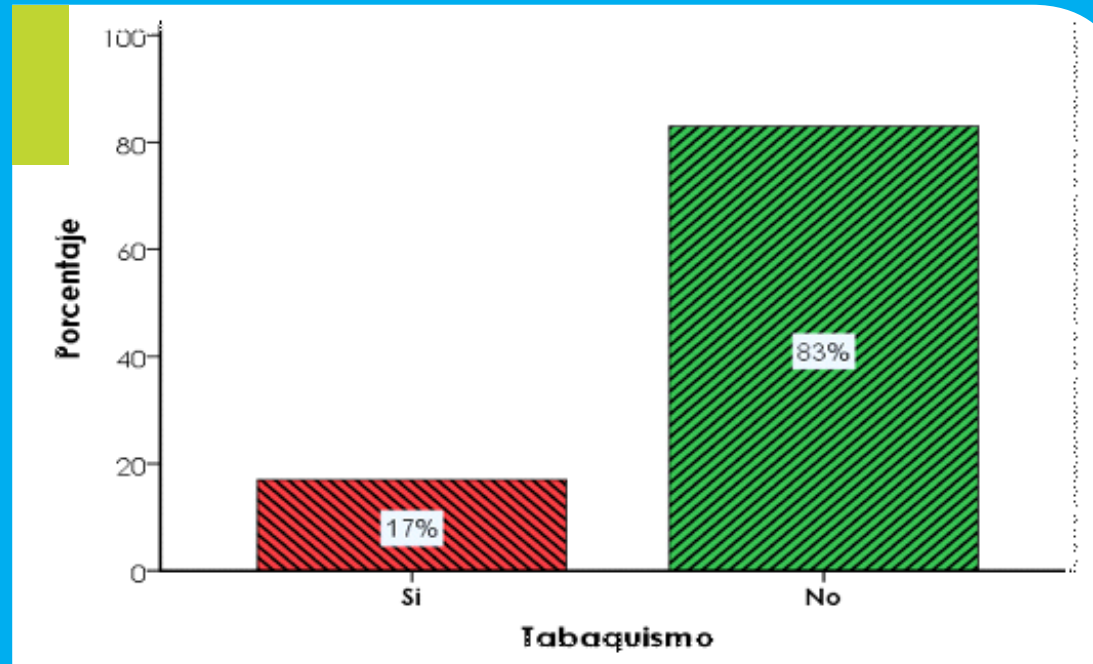

Gráfica 2 - Prácticas de tabaquismo en estudiantes de Licenciatura en Enfermería de la Universidad de Sonora del departamento de enfermería $(n=100$; fuente : encuesta directa) estudiantes de enfermería, que en su gran mayoría (60\%) come de 3 a 4 veces al día. Asimismo se valoró la salud bucal, el $60 \%$ se lava los dientes 3 veces o más al día después de cada comida. Se investigó también si los jóvenes utilizan medicamentos sin receta médica cuando tienen un problema de salud, mostrando que el $71 \%$ de la población total respondieron que "a veces" lo hacen, un $16 \%$ dijeron nunca hacerlo y el 13\% mencionó hacerlo de forma ocasional.

En el área de prevención de riesgos, al preguntar a los encuestados acerca de tener una vida sexual activa el $48 \%$ dijo abstenerse y gran parte de la población (52\%) respondió tener relaciones sexuales y utilizar como principal método de planificación familiar el preservativo (47\%). 


\section{GRAFICA 3}

Al analizar la frecuencia con la que los estudiantes acuden al médico, se obtuvo que en su gran mayoría (64\%) asisten a una consulta médica solo cuando se enferma y el $48 \%$ acude al dentista, pero solo asisten cuando tiene un problema dental.

\section{GRAFICA 4}

En la dimensión de prevención de riesgos, la frecuencia con la que se realizaban exámenes de salud médico-preventivo (por ejemplo: examen mamario y prueba de papanicolau en las mujeres y examen testicular en hombres) mostrando que en su gran mayoría (54\%) contestó practicarlo "a veces", 36 alumnos (36\%) reconoció nunca llevarlos a cabo y solo 10 alumnos de la población total (10\%) dijo realizarlos siempre.

\section{DISCUSIÓN}

En la teoría del autocuidado, parte de la base de que los individuos que realizan acciones de autocuidado son personas maduras o en proceso de maduración y que poseen las capacidades necesarias para cuidar de sí mismos. Por lo que se incluye en esta categoría a los jóvenes universitarios.

En su teoría de déficit de autocuidado describe a los individuos incapaces de conocer los requisitos para su propio autocuidado.

Con respecto a esto, estudios determinan que los estudiantes de enfermería poseen un déficit en áreas básicas de desarrollo tales como actividad, reposo y alimentación, encontrando similitudes en nuestra investigación debido a que la mitad de la población no realiza actividad física, más de la mitad (53\%) duerme menos de 6 horas así como tampoco

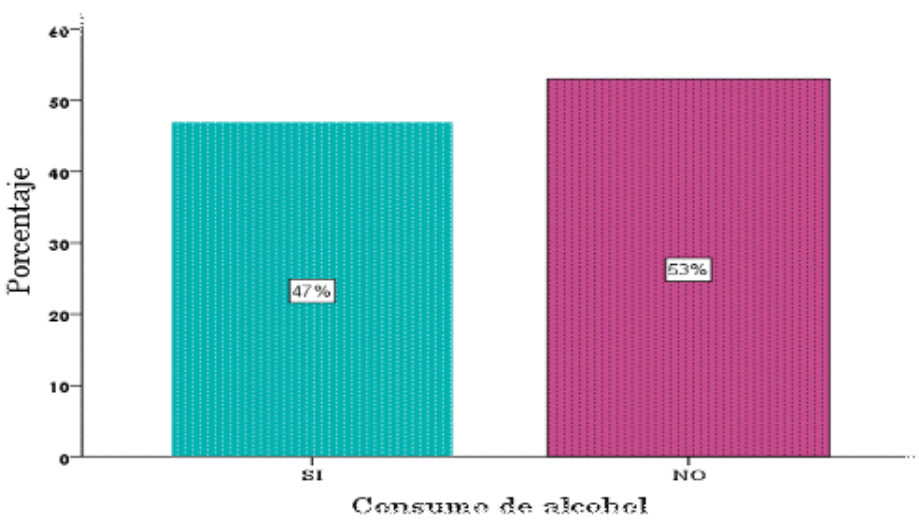

Gráfica 3 - Prácticas de consumo de alcohol de estudiantes de Licenciatura en Enfermería de la Universidad de Sonora del departamento de enfermería ( $\mathrm{n}=100$; fuente : encuesta directa)

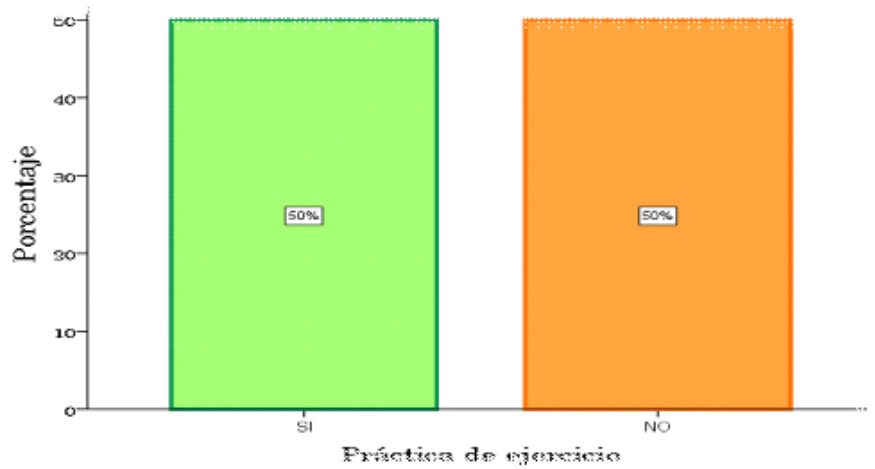

Gráfica 4 - Prácticas de ejercicio de estudiantes de Licenciatura en Enfermería de la Universidad de Sonora del departamento de enfermería ( $n=100$; fuente : encuesta directa)

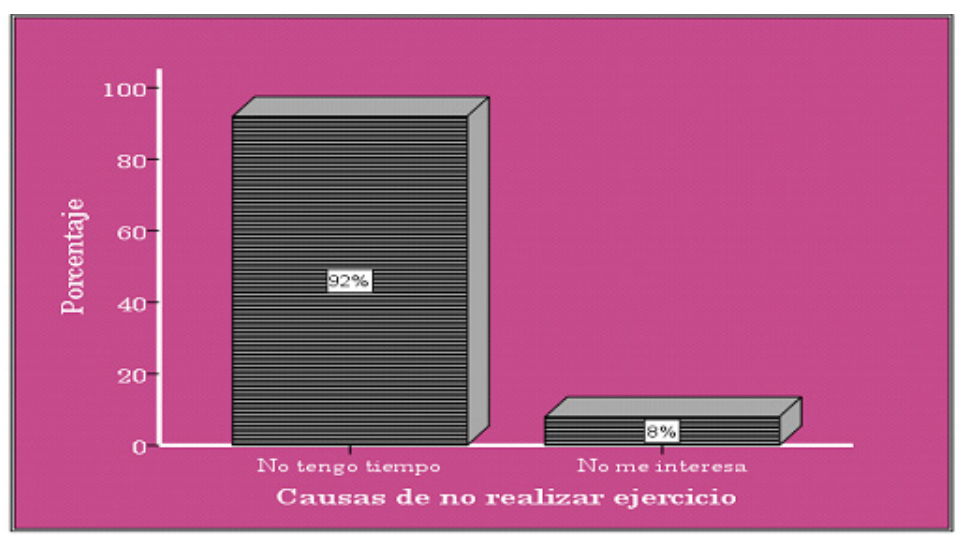

GRÁfICA 5 - Causas por las cuales no realizan ejercicio los estudiantes de Licenciatura en Enfermería de la Universidad de Sonora del departamento de enfermería ( $\mathrm{n}=100$; fuente: encuesta directa) 


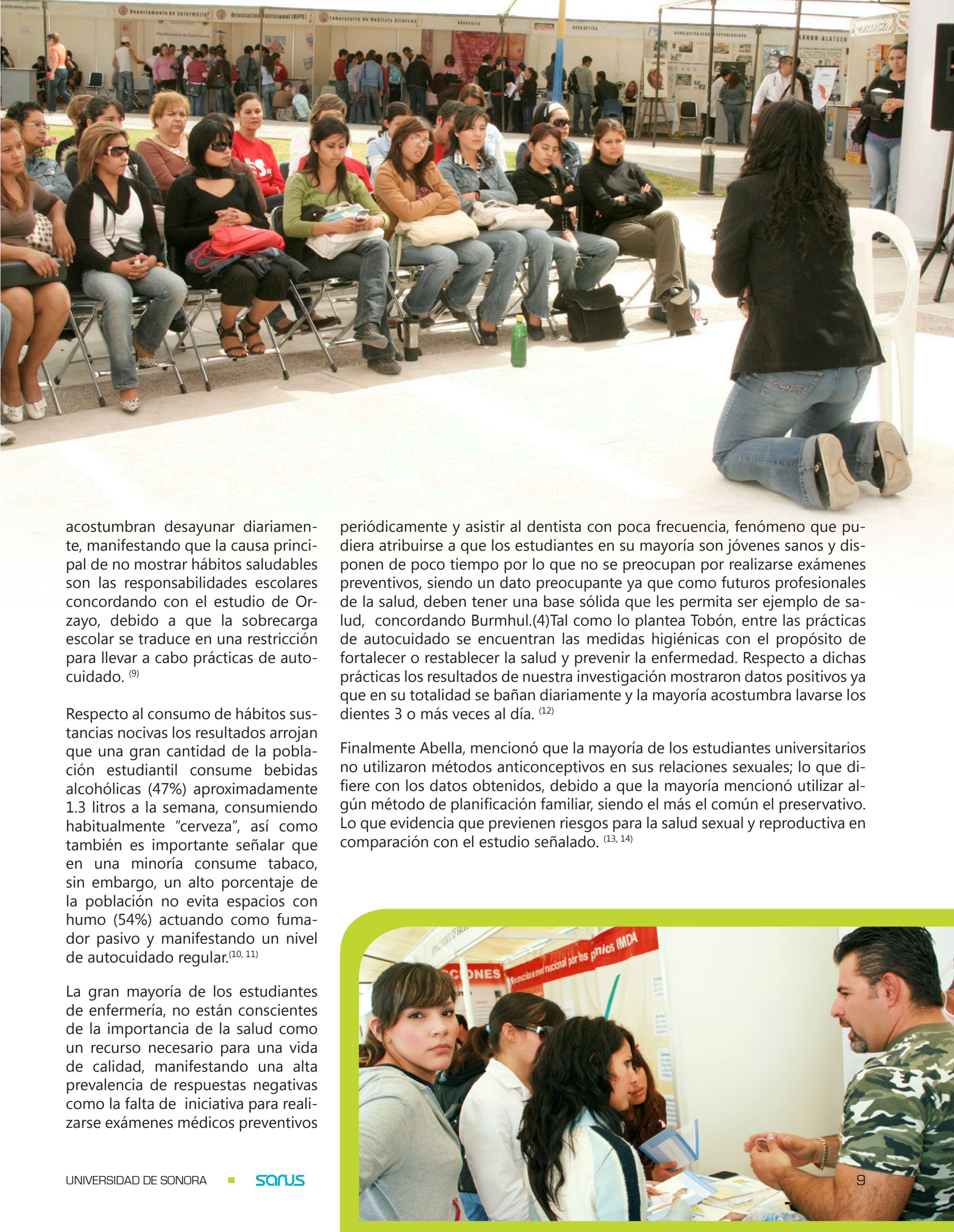


Estudios como el que se ha presentado ofrece la oportunidad para identificar hábitos con el fin de mejorar la calidad del autocuidado de los estudiantes de enfermería.

De acuerdo con los objetivos de este trabajo, la conclusión más relevante a la que podemos llegar es que la agencia de autocuidado puede verse afectada en su desarrollo por una serie de factores consideradas en déficit como la falta de tiempo para desayunar diariamente y realizar actividad física, la sobrecarga escolar que no les permite dormir adecuadamente así como también la automedicación cuando se tiene un problema de salud, esto combinado con falta de actitudes preventivas y falta de iniciativa para la realización de exámenes médicos.

La mayoría tuvo niveles bajos de consumo de tabaco pero presentan niveles altos de consumo de alcohol. Mencionaron adecuadas prácticas saludables, como ingesta suficiente de agua al día, buenos hábitos higiénicos, relaciones sexuales seguras y prevención de riesgos.

Del autocuidado se determina que en esta muestra el nivel es regular sin diferencias significativas entre sexos.

Se concluye que a nivel escuela se debe considerar el tiempo que los estudiantes deben dedicar a las labores académicas como parte de su plan de estudios y las horas que el alumno necesita para llevar a cabo prácticas saludables, que en éste estudio fueron consideradas en déficit y establecer un programa que favorezca los estilos de vida saludable y la organización del tiempo.

Se sugiere alentar a los estudiantes a acudir a los servicios de salud tales como consultorios médicos y de enfermería, asesorías nutricionales y exámenes médico-preventivos que ofrece la Universidad de Sonora, lo que permitirá formar profesionales de calidad que sean capaces de contribuir a una población más saludable.

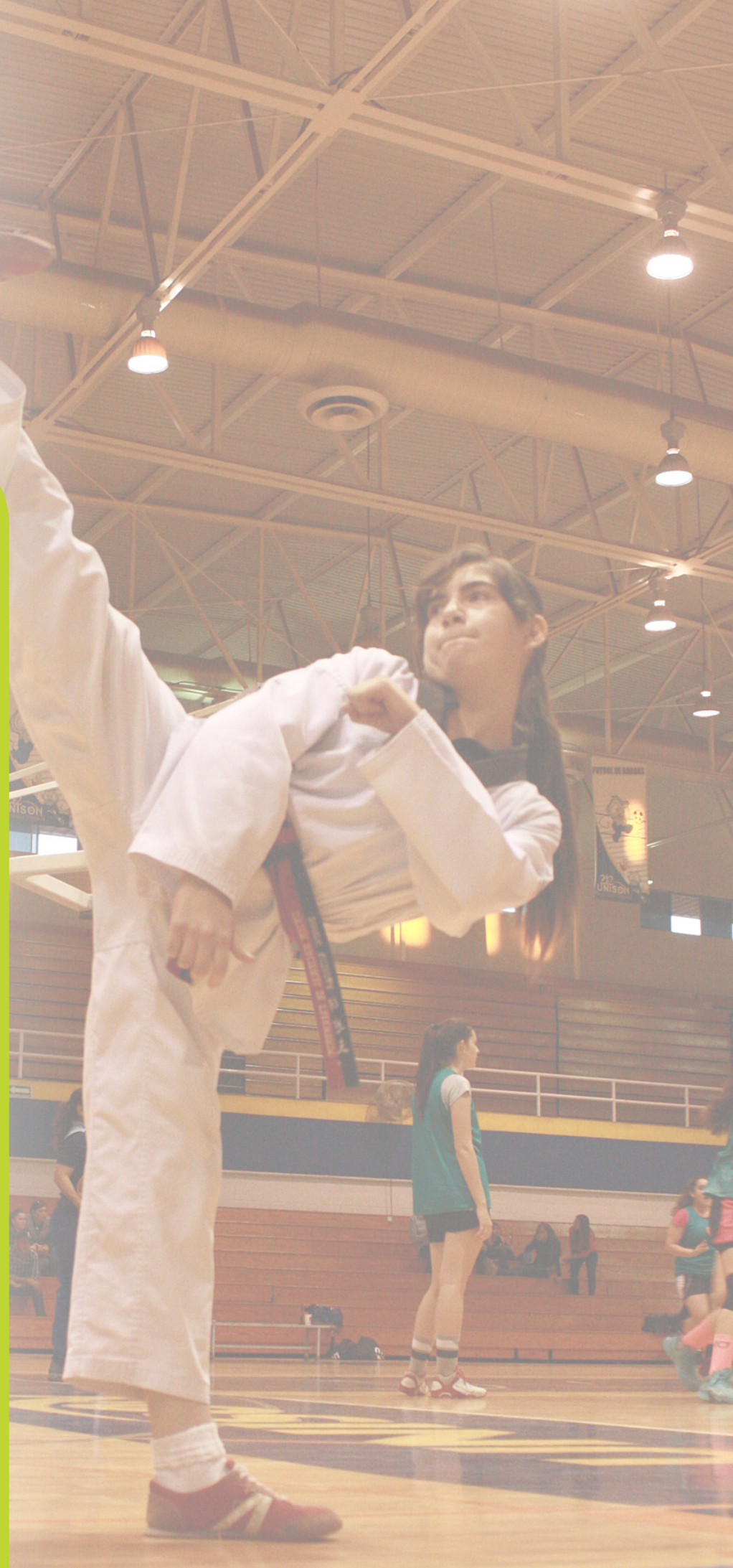




\section{REFERENCIAS BIBLIOGRÁFICAS}

1. Instituto Nacional de Estadística GeI. Instituto mexicano nacional de la juventud. 2010.

2. Juventud IMdL. Instituto mexicano de la juventud. 2005

3. Bañales FT, Quiles OL. El escenario sociocultural de los jóvenes en México. Dedica Revista de Educação e Humanidades. 2013 (4):239-55.

4. Burmuhl EC. Factores asosciados a la agencia de autocuidados de los estudiantes de 3 y 4 año de enfermeria. universidad de chile facultad de medicina , escuela de enfermeria. 2007:10,1-91.

5. Cuteliffe J. modelos de enfermeria, aplicacion a la practica. modelos de enfermeria, aplicacion a la practica. colombia: manual moderno; 2010. p. 155-77.

6. Alligood MR, Tomey AM. Modelos y teorías en enfermería: Elsevier Health Sciences Spain; 2011.

7. Burns N, Grove SK. Investigación en enfermería + Evolve: Desarrollo de la práctica enfermera basada en la evidencia: Elsevier Health Sciences Spain; 2012.

8. Daniel WW. Bioestadistica: Base para el analisis de las ciencias de la salud: Editorial Limusa S.A. De C.V.; 2002.

9. Oyarzo DPR. Autocuidado en los estudiantes de la carrera de Enfermería de la Universidad del Bío Bio: un análisis según el Modelo de Autocuidado de Dorothea Orem. 2010.

10. Hernández, Martínez D, Muñoz PS. Conductas de Autocuidado en estudiantes universitarios residentes del complejo de hogares Huachocopihue de la UACh, 2006. 2011.

11. Polo A, Hernández JM, Pozo C. Evaluación del estrés académico en estudiantes universitarios. Ansiedad y estrés. 1996;2(2-3):159-72.

12. Tobón Correa O. El autocuidado una habilidad para vivir. Hacia promoc salud. 2003 (8):37-49.

13. Abella Pons F, Farré Perisé Y, Caballer Fossas A, Adrover Villanueva À. Conductas de riesgo en jóvenes universitarios. 2005.

14. Espinoza L, Rodríguez F, Gálvez J, MacMillan N. Hábitos de alimentación y actividad física en estudiantes universitarios. Revista chilena de nutrición. 2011;38(4):458-65. 